\title{
GLOBAL CROSS SECTIONS FOR ANOSOV FLOWS
}

\author{
SLOBODAN N. SIMIĆ
}

\begin{abstract}
We provide a new criterion for the existence of a global cross section to a volumepreserving Anosov flow. The criterion is expressed in terms of expansion and contraction rates of the flow and is more general than the previous results of similar kind.
\end{abstract}

\section{INTRODUCTION}

Henri Poincaré introduced the idea of a cross section to a flow to study the 3-body problem. A global cross section to a flow $\Phi$ on a manifold $M$ is a codimension one submanifold $\Sigma$ of $M$ such that $\Sigma$ intersects every orbit of $\Phi$ transversely. It is natural to ask whether any given non-singular flow admits one.

If $\Sigma$ is a global cross section for $\Phi$, it is not hard to check that every orbit which starts on $\Sigma$ returns to $\Sigma$ after some positive time, defining the Poincaré first-return map $g: \Sigma \rightarrow \Sigma$. The analysis of $\Phi$ can then be reduced to the study of the map $g$, which in principle can be an easier task. The flow can be reconstructed from the Poincaré map by suspending it (cf., [KH95]).

The object of this paper is to investigate the existence of global cross sections to volume-preserving Anosov flows.

Recall that a non-singular flow $\Phi=\left\{f_{t}\right\}$ on a closed (compact and without boundary) Riemannian manifold $M$ is called Anosov if there exists an invariant splitting $T M=E^{s s} \oplus E^{c} \oplus E^{u u}$ of the tangent bundle of $M$ and uniform constants $c>0,0<\mu_{-} \leq \mu_{+}<1$ and $\lambda_{+} \geq \lambda_{-}>1$ such that the center bundle $E^{c}$ is spanned by the infinitesimal generator $X$ of the flow and for all $v \in E^{s s}, w \in E^{u u}$, and $t \geq 0$, we have

$$
\frac{1}{c} \mu_{-}^{t}\|v\| \leq\left\|T f_{t}(v)\right\| \leq c \mu_{+}^{t}\|v\|
$$

and

$$
\frac{1}{c} \lambda_{-}^{t}\|w\| \leq\left\|T f_{t}(v)\right\| \leq c \lambda_{+}^{t}\|w\|
$$

where $T f_{t}$ denotes the derivative (or tangent map) of $f_{t}$. We call $E^{s s}$ and $E^{u u}$ the strong stable and strong unstable bundles; $E^{c s}=E^{c} \oplus E^{s s}$ and $E^{c u}=E^{c} \oplus E^{u u}$ are called the center stable and center unstable bundles. It is well-known [HPS77, Has94] that all of them are Hölder continuous and uniquely integrable [Ano67]. The corresponding foliations will be denoted by $W^{s s}, W^{u u}, W^{c s}$, and $W^{c u}$. They are also Hölder continuous in the sense that each one admits Hölder foliation charts. This means that if $W^{\sigma}(\sigma \in\{s s, u u, c s, c u\})$ is $C^{\theta}$, then every point in $M$ lies in a $C^{\theta}$ chart $(U, \varphi)$ such that in $U$ the local $W^{\sigma}$-leaves are given by $\varphi_{k+1}=$ constant, $\ldots, \varphi_{n}=$ constant, where $\varphi=\left(\varphi_{1}, \ldots, \varphi_{n}\right)$ is a $C^{\theta}$ homeomorphism and $k$ is the dimension of $W^{\sigma}$. The leaves of all invariant foliations are as smooth as the flow. See also [PSW97] for a discussion of regularity of Hölder foliations.

Date: October 12, 2018. 
Related work. The first results on the existence of global cross sections to Anosov flows were proved by Plante in [Pla72]. He showed that if $E^{s s} \oplus E^{u u}$ is a uniquely integrable distribution or equivalently, if the foliations $W^{s s}$ and $W^{u u}$ are jointly integrable ${ }^{1}$, then the Anosov flow admits a global cross section. Sharp [Sha93] showed that a transitive Anosov flow admits a global cross section if it is not homologically full; this means that for every homology class $\alpha \in H_{1}(M, \mathbb{Z})$ there is a closed $\Phi$-orbit $\gamma$ whose homology class equals $\alpha$. (This is equivalent to the condition that there is no fully supported $\Phi$-invariant ergodic probability measure whose asymptotic cycle in the sense of Schwartzman [Sch57] is trivial.) Along different lines Bonatti and Guelman [BG09] showed that if the time-one map of an Anosov flow can be $C^{1}$ approximated by Axiom A diffeomorphisms, then the flow is topologically equivalent to the suspension of an Anosov diffeomorphism.

Let $n_{s}=\operatorname{dim} E^{s s}$ and $n_{u}=\operatorname{dim} E^{u u}$. If $n_{u}=1$ or $n_{s}=1$, the Anosov flow is said to be of codimension one. In the discussion that follows we always assume $n_{u}=1$. In [Ghy89] Ghys proved the existence of global cross sections for codimension one Anosov flows in the following cases: (1) if $E^{s u}=E^{s s} \oplus E^{u u}$ is $C^{1}$ and $n \geq 4$ (in this case the global cross section has constant return time); if (2) the flow is volume-preserving, $n \geq 4$ and $W^{c s}$ is of class $C^{2}$. This was generalized by the author in [Sim96] and [Sim97] where we showed that a codimension one Anosov flow admits a global cross section if any of the following assumptions is satisfied: (1) $E^{s u}$ is Lipschitz (in the sense that it is locally spanned by Lipschitz vector fields) and $n \geq 4$; (2) the flow is volume-preserving, $n \geq 4$, and $E^{s u}$ is $C^{\theta}$-Hölder for all $\theta<1$ (3) the flow is volume-preserving, $n \geq 4$, and $E^{c s}$ is of class $C^{1+\theta}$ for all $\theta<1$. Note that all the regularity assumptions above require that the invariant bundles be smoother than they usually are: $E^{s u}$ is generically only Hölder continuous and in the codimension one case, $E^{c s}$ is generically only $C^{1+\theta}$ for some small $0<\theta<1$. See [Has94, Has97] and [HW99].

The goal of this paper is to establish the following result.

Theorem. Let $\Phi=\left\{f_{t}\right\}$ be a volume-preserving Anosov flow on a closed Riemannian manifold $M$ and let $0<\theta \leq 1$ be the smaller of the Hölder exponents of $W^{s s}$ and $W^{c s}$. If

$$
\mu_{+}^{\left(n_{s}-1\right) \theta} \lambda_{+}^{\left(n_{u}-1\right) \theta}<\mu_{-}^{2(1-\theta)},
$$

then $\Phi$ admits a global cross section.

Remarks. (a) The condition (1.3) has a chance of being satisfied only if $n_{u}$ is much smaller than $n_{s}$. If $n_{u}>n_{s}$, then by reversing time it is easy to show that

$$
\lambda_{+}^{2(1-\alpha)}<\lambda_{-}^{\left(n_{u}-1\right) \alpha} \mu_{-}^{\left(n_{s}-1\right) \alpha}
$$

also implies the existence of a global cross section, where $\alpha$ is the minimum of the Hölder exponents of $E^{u u}$ and $E^{c u}$.

(b) If the flow is of codimension one with $n_{u}=1$, then (1.3) reduces to

$$
\mu_{+}^{(n-3) \theta}<\mu_{-}^{2(1-\theta)} .
$$

It is well-known (cf., [Has94] and [HPS77]) that the center stable bundle $E^{c s}$ and strong unstable bundle $E^{u u}$ of a volume-preserving Anosov flow in dimensions $n \geq 4$ are both $C^{1+\text { Hölder }}$. Thus if $E^{s u}$ is Lipschitz as in [Sim96] or $C^{\theta}$, for all $\theta<1$, as in [Sim97], then (1.4) is clearly satisfied. If $E^{c s}$ is $C^{1+\theta}$ for all $\theta<1$ as in [Sim97], then it is not hard to show that $E^{s s}$ is necessarily of class $C^{\theta}$ for all $\theta<1$, which again implies (1.4). Therefore, in the case of volume-preserving codimension one Anosov flows, our result implies all the previously known criteria for the existence of global cross sections.

\footnotetext{
${ }^{1}$ This means that locally speaking the $W^{u u}$-holonomy between local $W^{c s}$-leaves takes local $W^{s s}$-leaves to $W^{s s}$ leaves.
} 
(c) In the early 1970's, prompted by a dearth of examples, A. Verjovsky conjectured [Ver74] that every codimension one Anosov flow in dimensions $n \geq 4$ admits a global cross section. The importance of Verjovsky's conjecture stems from the fact that codimension one Anosov diffeomorphisms were classified by Franks [Fra70] and Newhouse [New70] who showed that every such diffeomorphism is topologically conjugate to a linear hyperbolic automorphism of a torus. Therefore, the affirmation of Verjovsky's conjecture would yield a complete classification of codimension one Anosov flows in dimensions $n \geq 4$.

Progress towards Verjovsky's conjecture was made in the early 1980's by Plante [Pla81, Pla83] and Armendariz [Arm82] who showed that the conjecture holds if the fundamental group of the manifold is solvable. By the work of Asaoka [Asa08] it follows that it suffices to prove the conjecture for volume-preserving flows, since any topologically transitive codimension one Anosov flow is topologically equivalent to a volume-preserving one. As of this writing, the conjecture remains open.

Throughout this paper smooth will mean of class $C^{\infty}$.

Outline of the proof. The main idea of the proof of the theorem is to find a smooth closed 1-form $\eta$ such that $\eta(X)>0$, where $X$ is the infinitesimal generator of the Anosov flow. It is not hard to see that this immediately implies the existence of a global cross section (cf., §3). To construct $\eta$, we use the fact that for any $k$-form $\xi$, the $C^{0}$-distance from $\xi$ to the space of closed $k$-forms is bounded above by the $C^{0}$ norm $\|d \xi\|$; see Proposition 2.4. It therefore suffices to construct a smooth 1-form $\xi$ such that $\left|\xi\left(X_{p}\right)\right|>\|d \xi\|$, for all $p \in M$, since Proposition 2.4 then yields a smooth closed 1-form $\eta$ such that $\eta(X)>0$. We will actually construct a smooth 1-form $\xi$ such that $\xi(X)=1$ and $\|d \xi\|<1$.

The construction of $\xi$ is divided into two steps. In the first step, we find an initial candidate for $\xi$ such that the norm of its exterior derivative restricted to $E^{c s}$ is small, while its total norm blows up in a way controlled by the Hölder exponent $\theta$. More precisely, for each $\varepsilon>0$ we construct a smooth 1-form $\xi_{0}^{\varepsilon}$ on $M$ such that $\xi_{0}^{\varepsilon}(X)=1, \| d \xi_{0}^{\varepsilon}\left\lceil_{E^{c s}} \| \leq D \varepsilon^{\theta}\right.$, and $\left\|d \xi_{0}^{\varepsilon}\right\| \leq K \varepsilon^{\theta-1}$, where $D$ and $K$ are positive constants independent of $\varepsilon$. This is achieved by carefully building smooth local cross sections and the corresponding flow boxes; cf., $\S 2.2$.

In the second step, we pull back $\xi_{0}^{\varepsilon}$ by $f_{-t}$ for suitable $t>0$ to make the norm of its exterior derivative small in the remaining directions. For this, we use an estimate (see Lemma 2.5) on the growth of $\left\|T f_{-t}(v \wedge w)\right\|$, for $t>0, v \in E^{s s}$ and $w \in E^{u u}$. Assuming (1.3), we then show that there exist $\varepsilon>0$ and $t>0$ such that $\xi=f_{-t}^{*} \xi_{0}^{\varepsilon}$ has the desired properties.

\section{Preliminaries}

2.1. Regularization. Here we recall a standard technique for approximating locally integrable functions by smooth ones called regularization or mollification (see [Eva98, Ste70]). Define the standard mollifier $\eta: \mathbb{R}^{n} \rightarrow \mathbb{R}$ by

$$
\eta(x)= \begin{cases}A_{0} \exp \left(\frac{1}{|x|^{2}-1}\right) & \text { if }|x|<1 \\ 0 & \text { if }|x| \geq 1\end{cases}
$$

where $A_{0}$ is chosen so that $\int \eta d x=1$. For every $\varepsilon>0$, set $\eta_{\varepsilon}(x)=\varepsilon^{-n} \eta(x / \varepsilon)$. Note that the support of $\eta_{\varepsilon}$ is contained in the ball $B(0, \varepsilon)$ of radius $\varepsilon$ centered at the origin and that $\int \eta_{\varepsilon} d x=1$. 
For a locally integrable function $u: \mathbb{R}^{n} \rightarrow \mathbb{R}$ define

$$
u^{\varepsilon}(x)=\left(u * \eta_{\varepsilon}\right)(x)=\int_{\mathbb{R}^{n}} u(y) \eta_{\varepsilon}(x-y) d y=\int_{\mathbb{R}^{n}} u(x-y) \eta_{\varepsilon}(y) d y .
$$

2.1. Proposition. Assume that $u: \mathbb{R}^{n} \rightarrow \mathbb{R}$ is locally integrable. Then:

(a) $u^{\varepsilon} \in C^{\infty}\left(\mathbb{R}^{n}\right)$.

(b) If $u \in L^{\infty}$, then $\left\|u^{\varepsilon}\right\|_{L^{\infty}} \leq\|u\|_{L^{\infty}}$.

(c) If $u$ is continuous, then $u^{\varepsilon} \rightarrow u$, uniformly as $\varepsilon \rightarrow 0$. If $u \in C^{\theta}(0<\theta \leq 1)$, then $\left\|u^{\varepsilon}-u\right\|_{C^{0}} \leq\|u\|_{C^{\theta}} \varepsilon^{\theta}$, where the $C^{\theta}$-norm is the sum of its sup-norm and its best Hölder constant.

(d) If $u \in C^{\theta}$, then

$$
\left\|d u^{\varepsilon}\right\| \leq\|d \eta\|_{L^{1}}\|u\|_{C^{\theta}} \varepsilon^{\theta-1}
$$

where $\|d \eta\|_{L^{1}}=\max _{i} \int_{\mathbb{R}^{n}}\left|\partial \eta / \partial x_{i}\right| d x$ and $\left\|d u^{\varepsilon}\right\|$ denotes the maximum of the sup-norms of the partial derivatives of $u^{\varepsilon}$.

Proof. Proofs of (a), (b) and the first part of (c) can be found in [Eva98]. For the second part of (c), we have

$$
\begin{aligned}
\left|u^{\varepsilon}(x)-u(x)\right| & =\left|\int_{B(0, \varepsilon)} \eta_{\varepsilon}(y)[u(x-y)-u(x)] d y\right| \\
& \leq\|u\| \varepsilon^{\theta} \int_{B(0, \varepsilon)} \eta_{\varepsilon}(y) d y \\
& =\|u\|_{C^{\theta}} \varepsilon^{\theta} .
\end{aligned}
$$

If $u \in C^{1}$, then the same estimates hold with $\theta$ replaced by 1 .

Observe that since $\eta_{\varepsilon}$ has compact support,

$$
\int_{\mathbb{R}^{n}} \frac{\partial \eta_{\varepsilon}}{\partial x_{i}}(y) d y=0
$$

for $1 \leq i \leq n$. Note also that

Assuming $u \in C^{\theta}$, we obtain (d):

$$
\frac{\partial \eta_{\varepsilon}}{\partial x_{i}}(x)=\frac{1}{\varepsilon^{n+1}} \frac{\partial \eta}{\partial x_{i}}\left(\frac{x}{\varepsilon}\right)
$$

$$
\begin{aligned}
\left|\frac{\partial u^{\varepsilon}}{\partial x_{i}}(x)\right| & =\left|\int_{\mathbb{R}^{n}} u(x-y) \frac{\partial \eta_{\varepsilon}}{\partial x_{i}}(y) d y\right| \\
& \text { by } \stackrel{(2.1)}{=}\left|\int_{B(0, \varepsilon)}[u(x-y)-u(x)] \frac{\partial \eta_{\varepsilon}}{\partial x_{i}}(y) d y\right|, \\
& \leq\|u\|_{C^{\theta}} \varepsilon^{\theta} \int_{B(0, \varepsilon)}\left|\frac{\partial \eta_{\varepsilon}}{\partial x_{i}}(y)\right| d y \\
& =\|u\|_{C^{\theta}} \varepsilon^{\theta} \int_{B(0, \varepsilon)} \frac{1}{\varepsilon^{n+1}}\left|\frac{\partial \eta}{\partial x_{i}}\left(\frac{y}{\varepsilon}\right)\right| d y \\
& z=\frac{y}{\varepsilon}\|u\|_{C^{\theta}} \varepsilon^{\theta} \cdot \frac{1}{\varepsilon} \int_{B(0,1)}\left|\frac{\partial \eta}{\partial x_{i}}(z)\right| d z \\
& \leq\|d \eta\|_{L^{1}}\|u\|_{C^{\theta}} \varepsilon^{\theta-1} .
\end{aligned}
$$


2.2. Corollary. Let $M$ be a compact manifold without boundary. Fix a finite atlas $\mathscr{A}=\left\{\left(U_{i}, \varphi_{i}\right)\right.$ : $i \in I\}$ of $M$. If $u: U \rightarrow \mathbb{R}$ is $C^{\theta}$ and $U \subset U_{j}$ for some $j \in I$, then there exist $\varepsilon_{*}>0$ and a family of smooth approximations $u^{\varepsilon}\left(0<\varepsilon<\varepsilon_{*}\right)$ of $u$ such that:

(a) $u^{\varepsilon}$ is defined on $U^{\varepsilon} \subset U$ where $U^{\varepsilon}=\varphi_{j}^{-1}\left\{x \in \varphi_{j}(U): d\left(x, \partial \varphi_{j}(U)\right)>\varepsilon\right\}$.

(b) $\left\|u^{\varepsilon}-u\right\|_{C^{0}} \leq \kappa \varepsilon^{\theta}$.

(c) $\left\|d u^{\varepsilon}\right\|_{C^{0}} \leq \kappa \varepsilon^{\theta-1}$.

Here $\kappa>0$ depends only on $\mathscr{A}$ and $\|u\|_{C^{\theta}}$.

Proof. The family $u^{\varepsilon}=\left[\left(u \circ \varphi_{j}^{-1}\right) * \eta_{\varepsilon}\right] \circ \varphi_{j}$ has the desired properties. We can take $\varepsilon_{*}$ to be any positive number such that for $\varepsilon<\varepsilon_{*}$, the sets $U^{\varepsilon}$ defined above are non-empty.

2.2. Construction of local cross sections. In this section we construct a finite covering of the manifold by smooth flow boxes defined by carefully chosen smooth local cross sections.

Let $p \in M$ be arbitrary and choose a $W^{s s}$-foliation chart $(U, \varphi)$ containing $p$. This means that $\varphi: U \rightarrow \mathbb{R}^{n}$ is a $C^{\theta}$-homeomorphism such that the local $W^{s s}$-leaves in $U$ are given by

$$
\varphi_{n_{s}+1}=\text { constant }, \cdots, \varphi_{n}=\text { constant, }
$$

where $\varphi=\left(\varphi_{1}, \ldots, \varphi_{n}\right)$. We can also arrange that the local $W^{c s}$-leaves in $U$ are defined by

$$
\varphi_{n_{s}+1}=\text { constant }, \cdots, \varphi_{n-1}=\text { constant, }
$$

so that in each local $W^{c s}$-leaf in $U W^{s s}$ is given by $\varphi_{n}=$ constant. Furthermore, we can take $U$ so that its closure is contained in a smooth chart for $M$. This condition will allow us to mollify continuous functions defined on $U$ without having to shrink the domain.

Even though $\varphi$ is only Hölder, the flow invariance of $W^{s s}$ implies that each $\varphi_{i}$ is differentiable with respect to $X$. Since $X$ is tangent to the $W^{c s}$ leaves, it follows that $X \varphi_{i}=0$, for $n_{s}+1 \leq i \leq n-1$. Furthermore, the restriction of $W^{s s}$ to $W^{c s}$-leaves is as smooth as the flow, so $\varphi_{n}$ is smooth on the local $W^{c s}$-leaves in $U$. Since $X$ is uniformly transverse to $W^{s s}$ it is clear that $X \varphi_{n} \neq 0$ and by continuity there exists $\delta>0$ such that $\left|X \varphi_{n}\right| \geq \delta$ on $U$.

Define

$$
\Sigma_{0}=\bigcup_{x \in W_{\text {loc }}^{u u}(p)} W_{\text {loc }}^{s s}(x)
$$

where $W_{\text {loc }}^{\sigma}(x)$ denotes the local $W^{\sigma}$-leaf in $U$ (for $\sigma \in\{u u, s s\}$ ). Then $\Sigma_{0}$ is a Hölder continuous local cross section for the flow. (This makes sense, since the intersection of $\Sigma_{0}$ with each local $W^{c s}$-leaf is a local $W^{s s}$-leaf, hence smooth and transverse to the flow.) Let $\Sigma$ be a slightly smaller compact subset of $\Sigma_{0}$ containing $p$.

We claim that there exists $T>0$, depending on $\Sigma$ and $U$, such that

$$
V \stackrel{\text { def }}{=} \bigcup_{|t|<T} f_{t}(\Sigma)
$$

is a Hölder continuous flow box contained in $U$. Assume the contrary; it follows that the map $(t, q) \mapsto f_{t}(q)$ fails to be $1-1$ on $(-T, T) \times \Sigma$, for any $T>0$. Thus there exist sequences $\left(q_{k}\right)$ and $\left(T_{k}\right)$ such that $q_{k} \in \Sigma, T_{k}>0, T_{k} \rightarrow 0$ and $f_{T_{k}}\left(q_{k}\right) \in \Sigma$, for all $k$. Since $f_{T_{k}}\left(q_{k}\right)$ and $q_{k}$ lie in the same local $W^{c s}$-leaf, it follows that $\varphi_{n}\left(f_{T_{k}}\left(q_{k}\right)\right)=\varphi_{n}\left(q_{k}\right)$. On the other hand, by compactness of $\Sigma$, $\left(q_{k}\right)$ has a subsequence $\left(q_{k_{j}}\right)$ which converges to some $q \in \Sigma_{0}$. This implies $X \varphi_{n}(q)=0$, which contradicts $\left|X \varphi_{n}\right| \geq \delta$. Therefore, $T$ exists; we will call it the length of the continuous flow box $V$ (although a more appropriate name would be half-length). 
Define $\tau: V \rightarrow \mathbb{R}$ by

$$
\tau\left(f_{t} q\right)=t
$$

for $q \in \Sigma$ and $|t|<T$. It is clear that $\tau$ is $C^{\theta}, X \tau=1$ and $\tau$ is constant on the local $W^{s s}$-leaves in $V$.

Next we approximate $\Sigma, \tau$ and $V$ by smooth objects with similar properties.

2.3. Lemma. There exists $\varepsilon_{*}>0$ such that for every $0<\varepsilon<\varepsilon_{*}$ there exist an open set $V^{\varepsilon} \subset V$ and a smooth function $\tau^{\varepsilon}: V^{\varepsilon} \rightarrow \mathbb{R}$ with the following properties:

(a) $X \tau^{\varepsilon}=1$.

(b) $\left|d_{q} \tau^{\varepsilon}(v)\right| \leq A \varepsilon^{\theta}\|v\|$, for all $q \in V^{\varepsilon}$ and $v \in E^{s s}$, where $A$ is a constant independent of $\varepsilon, q$, and $v$.

(c) $\left\|d \tau^{\varepsilon}\right\| \leq B \varepsilon^{\theta-1}$, where $B$ is a constant independent of $\varepsilon$.

(d) The Hausdorff distance between $V$ and $V^{\varepsilon}$ tends to zero, as $\varepsilon \rightarrow 0$.

Proof. For the sake of notational simplicity, we will write $f(x) \lesssim g(x)(x \in S)$ to mean that there exists a constant $C$ independent of $x \in S$ such that $f(x) \leq C g(x)$, for all $x \in S$.

Since $U$ is contained in a smooth chart for $M$, by Corollary 2.2 there exists $\varepsilon_{*}$ such that for each $i>n_{s}$ there is a family $\varphi_{i}^{\varepsilon}\left(0<\varepsilon<\varepsilon_{*}\right)$ of smooth approximations of $\varphi_{i}$ satisfying

$$
\left\|\varphi_{i}^{\varepsilon}-\varphi_{i}\right\|_{C^{0}} \lesssim \varepsilon^{\theta} \quad \text { and } \quad\left\|d \varphi_{i}^{\varepsilon}\right\|_{C^{0}} \lesssim \varepsilon^{\theta-1} .
$$

Note that $u^{\varepsilon}$ is defined on all of $U$. Denote by $\mathscr{F}_{\varepsilon}$ the foliation of $U^{\varepsilon}$ defined by

$$
\varphi_{n_{s}+1}^{\varepsilon}=\text { constant }, \cdots, \varphi_{n}^{\varepsilon}=\text { constant. }
$$

It is easy to see that $\mathscr{F}_{\varepsilon}$ is smooth and the (largest principal) angle between $W_{\text {loc }}^{s s}$ and $\mathscr{F}_{\varepsilon}$ is $\lesssim \varepsilon^{\theta}$. Let

$$
\Sigma^{\varepsilon}=\bigcup_{x \in W_{\mathrm{loc}}^{u u}(p)} \mathscr{F}_{\varepsilon}(x) .
$$

Then $\Sigma^{\varepsilon}$ is a smooth local cross section and there exists $T_{\varepsilon}>0$ such that the set

$$
V^{\varepsilon}=\bigcup_{|t|<T_{\varepsilon}} f_{t}\left(\Sigma^{\varepsilon}\right)
$$

is a smooth flow box for $X$ contained in $U^{\varepsilon}$. It is clear that the length $T_{\varepsilon}$ of $V^{\varepsilon}$ is close to the length $T$ of $V$; we can also take $T_{\varepsilon} \leq T$.

Define $\tau^{\varepsilon}: V^{\varepsilon} \rightarrow \mathbb{R}$ by

$$
\tau^{\varepsilon}\left(f_{t} q\right)=t
$$

for all $q \in \Sigma^{\varepsilon}$ and $-T_{\varepsilon}<t<T_{\varepsilon}$. Clearly, $\tau^{\varepsilon}$ is smooth and $X \tau^{\varepsilon}=1$, proving (a).

We will first show that (b) holds along $\Sigma^{\varepsilon}$. Let $q \in \Sigma^{\varepsilon}$ be arbitrary. Since the angle between $\mathscr{F}_{\varepsilon}(q)$ and $W_{\text {loc }}^{s s}(q)$ is $\lesssim \varepsilon^{\theta}$, it follows that the angle between $\Sigma^{\varepsilon} \cap W_{\text {loc }}^{c s}(q)$ and $W_{\text {loc }}^{s s}(q)$ is also $\lesssim \varepsilon^{\theta}$. See Figure 1. Then the fact that $d \tau^{\varepsilon}=0$ on $\Sigma^{\varepsilon} \cap W_{\text {loc }}^{c s}(q)$ implies $\left\|d_{q} \tau^{\varepsilon} \uparrow_{E^{s s}}\right\| \lesssim \varepsilon^{\theta}$, as desired. To extend this to all points in $V^{\varepsilon}$ we will use the invariance of $d \tau^{\varepsilon}$ with respect to the flow: $f_{t}^{*}\left(d \tau^{\varepsilon}\right)=d \tau^{\varepsilon}$, whenever both sides are defined. For $q \in \Sigma^{\varepsilon},|t|<T_{\varepsilon}$ and $v \in E_{f_{t} q}^{s s}$, we have

$$
\begin{aligned}
\left|d_{f_{t} q} \tau^{\varepsilon}(v)\right| & =\left|d_{q} \tau^{\varepsilon}\left(T f_{-t}(v)\right)\right| \\
& \leq\left\|\left.d_{q} \tau^{\varepsilon}\right|_{E^{s s}}\right\|\left\|T f_{-t}(v)\right\| \\
& \lesssim \varepsilon^{\theta} \mu_{-}^{-t}\|v\| \\
& \leq \varepsilon^{\theta} \mu_{-}^{-T_{\varepsilon}}\|v\| \\
& \leq \varepsilon^{\theta} \mu_{-}^{-T}\|v\|,
\end{aligned}
$$




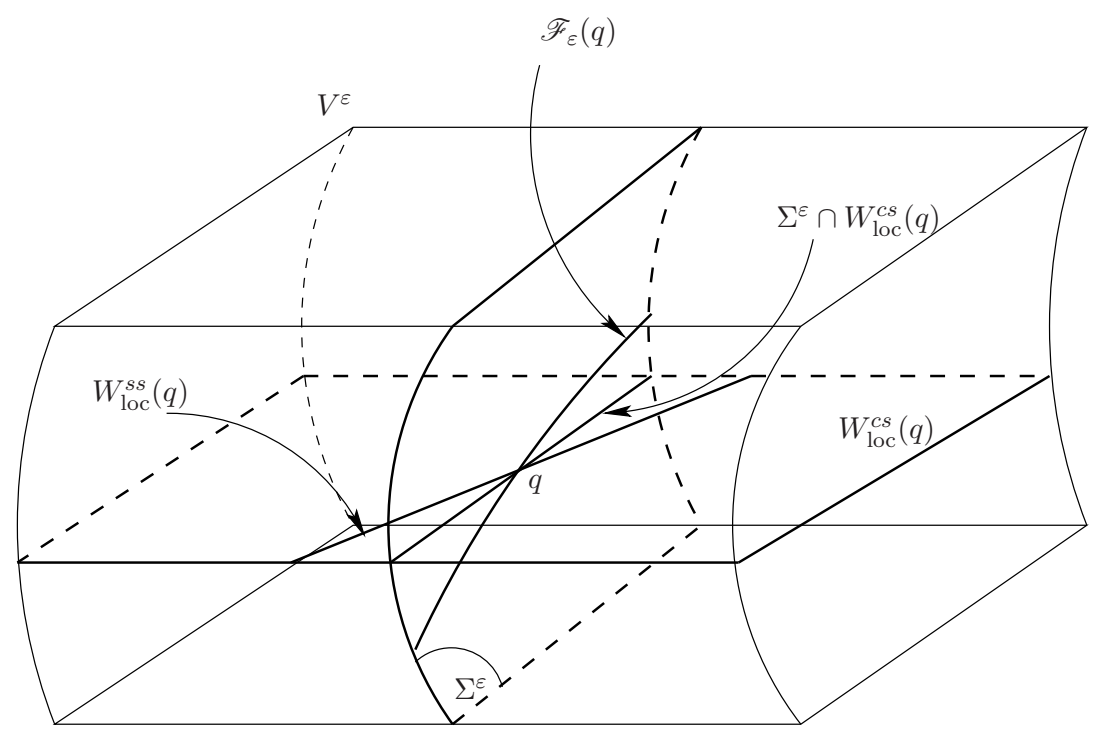

Figure 1. The flow box $V^{\varepsilon}$ with local cross section $\Sigma^{\varepsilon}$.

where we used $T_{\varepsilon} \leq T$. This proves (b) for all $q \in V^{\varepsilon}$.

To prove (c), we work in a smooth coordinate system in which $X=\partial / \partial x_{1}$. Since $\Sigma$ is a $C^{\theta}$ hypersurface, it is locally the graph of a $C^{\theta}$ function $g$ such that $\tau(g(z), z)=0$, for all $z$ is some open set in $\mathbb{R}^{n-1}$. Similarly, $\Sigma^{\varepsilon}$ is locally the graph of a smooth function $g^{\varepsilon}$ such that $\tau^{\varepsilon}\left(g^{\varepsilon}(z), z\right)=0$, for all $z$ in some open set in $\mathbb{R}^{n-1}$. Since $g^{\varepsilon} \rightarrow g$, as $\varepsilon \rightarrow 0$, (2.2) forces $\left\|d g^{\varepsilon}\right\| \lesssim \varepsilon^{\theta-1}$. Differentiating $\tau^{\varepsilon}\left(g^{\varepsilon}(z), z\right)=0$ with respect to $z_{i}$ for $i>1$ and using $\partial \tau^{\varepsilon} / \partial z_{1}=X \tau^{\varepsilon}=1$, we obtain

$$
\left|\frac{\partial \tau^{\varepsilon}}{\partial z_{i}}\right|=\left|\frac{\partial g^{\varepsilon}}{\partial z_{i}}\right| \lesssim \varepsilon^{\theta-1}
$$

Thus (c) holds on $\Sigma^{\varepsilon}$; we can extend it to $V^{\varepsilon}$ using the flow invariance of $d \tau^{\varepsilon}$ as in the proof of (b).

Part (d) holds by construction.

By the above analysis and compactness, we can cover $M$ by finitely many Hölder flow boxes $V_{1}, \ldots, V_{\ell}$, each of which is equipped with a local Hölder cross section $\Sigma_{i}$. We can approximate each $\Sigma_{i}$ by a smooth cross section $\Sigma_{i}^{\varepsilon}$ as above and obtain smooth flow boxes $V_{i}^{\varepsilon} \subset V_{i}$ and smooth functions $\tau_{i}^{\varepsilon}: V_{i}^{\varepsilon} \rightarrow \mathbb{R}$ satisfying the properties from Lemma 2.3; namely,

$$
X \tau_{i}^{\varepsilon}=1, \quad\left\|d \tau_{i}^{\varepsilon} \uparrow_{E^{s s}}\right\| \leq A_{i} \varepsilon^{\theta}, \quad\left\|d \tau_{i}^{\varepsilon}\right\| \leq B_{i} \varepsilon^{\theta-1},
$$

where the constants $A_{i}, B_{i}$ are independent of $\varepsilon$. Let $A=\max A_{i}, B=\max B_{i}$ (not to be confused with the constants $A, B$ in Lemma 2.3). By Lemma 2.3 (d), there exists $\varepsilon_{*}>0$ such that for all $0<\varepsilon<\varepsilon_{*}$ the sets $V_{1}^{\varepsilon}, \ldots, V_{\ell}^{\varepsilon}$ cover $M$.

2.3. Distance to the space of closed forms. We will consider $C^{r}$ differential $k$-forms $\xi$, with $r \geq 1$. We denote the $C^{0}$ norm of $\xi$ on $M$ by $\|\xi\|$ :

$$
\|\xi\|=\sup _{p \in M}\left|\xi_{p}\right|
$$

where $\left|\xi_{p}\right|$ is is the operator norm of $\xi_{p}$ as a $k$-linear map $T_{p} M \times \cdots \times T_{p} M \rightarrow \mathbb{R}$. 
Consider first a differential form $\omega$ on $M \times[0,1]$, where $t$ is the coordinate in $[0,1]$. Denote by $\pi_{M}: M \times[0,1] \rightarrow M$ and $\pi_{I}: M \times[0,1] \rightarrow[0,1]$ the obvious projections. Since

$$
T_{(p, t)}(M \times[0,1])=T_{p} M \oplus T_{t}[0,1],
$$

any differential $k$-form on $M \times[0,1]$ can be uniquely written as

$$
\omega=\omega_{0}+d t \wedge \eta
$$

where $\omega_{0}\left(v_{1}, \ldots, v_{k}\right)=0$ if some $v_{i}$ is in the kernel of $\left(\pi_{M}\right)_{*}$ and $\eta$ is a $(k-1)$-form with the analogous property (i.e., $i_{v} \omega_{0}=i_{v} \eta=0$, for every "vertical" vector $v \in T(M \times[0,1])$, where $i_{v}$ denotes contraction by $v)$.

Define a $(k-1)$-form $\mathscr{H}(\xi)$ on $M$ by

$$
\mathscr{H}(\omega)_{p}=\int_{0}^{1} j_{t}^{*} \eta_{(p, t)} d t
$$

where $j_{t}: M \rightarrow M \times[0,1]$ is defined by $j_{t}(p)=(p, t)$. It is well-known (cf., [Spi05]) that

$$
j_{1}^{*} \omega-j_{0}^{*} \omega=d(\mathscr{H} \omega)+\mathscr{H}(d \omega) .
$$

Considering $\mathscr{H}$ as a linear operator from $\Omega^{k}(M \times[0,1])$ to $\Omega^{k-1}(M)$, both equipped with the $C^{0}$ norm, it is not hard to see that

$$
\|\mathscr{H}\|=1 \text {. }
$$

We claim:

2.4. Proposition. Let $\xi$ be a $C^{r}$ differential $k$-form $(r \geq 1)$ on a closed manifold $M$. Then

$$
\inf \left\{\|\xi-\eta\|: \eta \in C^{r}, d \eta=0\right\} \leq\|d \xi\| .
$$

In other words, $\|d \xi\|$ is an upper bound on the distance from $\xi$ to the space of closed forms. The inequality also holds for continuous forms which admit a continuous exterior derivative.

Proof of the Proposition. First, let us show that the result holds on any manifold $M$ which is smoothly contractible to a point $p_{0}$ via $H: M \times[0,1] \rightarrow M$, where $H(p, 0)=p_{0}$ and $H(p, 1)=p$, for all $p \in M$. Since $H \circ j_{1}$ is the identity map of $M$ and $H \circ j_{0}$ is the constant map $p_{0}$, it follows that

$$
\xi=\left(H \circ j_{1}\right)^{*} \xi=j_{1}^{*}\left(H^{*} \xi\right) \quad \text { and } \quad 0=\left(H \circ j_{0}\right)^{*} \xi=j_{0}^{*}\left(H^{*} \xi\right) .
$$

Applying (2.5) to $H^{*} \xi$, we obtain

$$
\begin{aligned}
\xi & =\xi-0 \\
& =j_{1}^{*}\left(H^{*} \xi\right)-j_{0}^{*}\left(H^{*} \xi\right) \\
& =d \mathscr{H}(\xi)+\mathscr{H}(d \xi) .
\end{aligned}
$$

Using (2.6), we obtain

$$
\|\xi-d \mathscr{H}(\xi)\|=\|\mathscr{H}(d \xi)\| \leq\|d \xi\| .
$$

Therefore, the statement of the theorem holds for contractible $M$.

Let $M$ now be any closed manifold and $\xi$ a $C^{r} k$-form on $M, r \geq 1$. Cover $M$ by contractible open sets $U_{1}, \ldots, U_{m}$. Denote the operator $\mathscr{H}$ restricted to forms on $U_{i}$ by $\mathscr{H}_{i}$ and let $\xi_{i}$ be the restriction of $\xi$ to $U_{i}$. Define a $k$-form $\eta$ on $M$ by requiring that the restriction of $\eta$ to $U_{i}$ be equal to $d \mathscr{H}_{i}\left(\xi_{i}\right)$. We claim that $\eta$ is well-defined and closed. 
Indeed, $\xi_{i}=\xi_{j}$ on $U_{i} \cap U_{j}$, and $\mathscr{H}_{i}(\omega)=\mathscr{H}_{j}(\omega)$ for every $k$-form $\omega$ defined on $\left(U_{i} \cap U_{j}\right) \times[0,1]$. Thus on $U_{i} \cap U_{j}$, we have $d \mathscr{H}_{i}\left(\xi_{i}\right)=d \mathscr{H}_{j}\left(\xi_{j}\right)$, so $\eta$ is well-defined. Since $\eta$ is locally exact, it follows that it is closed. By (2.4), we obtain

$$
\|\xi-\eta\| \leq \max _{1 \leq i \leq m}\left\|\xi_{i}-d \mathscr{H}_{i}\left(\xi_{i}\right)\right\| \leq \max _{1 \leq i \leq m}\left\|d \xi_{i}\right\| \leq\|d \xi\|
$$

This completes the proof of the proposition.

2.4. Change of Riemannian metric. Denote by $\Omega$ the smooth volume form preserved by the flow and let $\mathscr{R}$ be the Riemannian metric which induces $\Omega$.

Our goal is to show that relative to some Riemannian metric the area of the parallelogram $T f_{-t}(v \wedge w)$ grows as $\left(\mu_{+}^{n_{s}-1} \lambda_{+}^{n_{u}-1}\right)^{t}$, where $v \in E^{s s}, w \in E^{u u}$, and $t \geq 0$. To do this, it will be convenient to switch from the original Riemannian metric $\mathscr{R}$ to a new metric $\mathscr{R}^{\prime}$ with respect to which $X$ is a unit vector and $E^{c} \oplus E^{s s} \oplus E^{u u}$ is an orthogonal splitting. This metric can in general be only continuous and the corresponding volume form $\Omega^{\prime}$ may not be invariant with respect to the flow. We will show that this does not present a problem.

Let $\mathscr{R}^{\prime}$ be as above and let $\Omega^{\prime}$ be the Riemannian volume form induced by $\mathscr{R}^{\prime}$. Since $\Omega$ and $\Omega^{\prime}$ are both volume forms, there exists a positive continuous function $\phi$ such that $\Omega^{\prime}=\phi \Omega$. Let

$$
L=\frac{\max _{M} \phi}{\min _{M} \phi} .
$$

Denote the norms of tangent vectors (and their wedge products) with respect to $\mathscr{R}$ and $\mathscr{R}^{\prime}$ by $\|\cdot\|$ and $\|\cdot\|^{\prime}$, respectively. By compactness of $M$ there exist $b_{-}, b_{+}>0$ such that

$$
b_{-}\|v\| \leq\|v\|^{\prime} \leq b_{+}\|v\|
$$

for all $v \in T M$. Observe that for $v \in E^{s s}, w \in E^{u u}$, and $t \geq 0$, we have

$$
\left\|T f_{t}(v)\right\|^{\prime} \leq b c \mu_{+}^{t}\|v\|^{\prime} \quad \text { and } \quad\left\|T f_{t}(w)\right\|^{\prime} \leq b c \lambda_{+}^{t}\|w\|^{\prime},
$$

where $b=b_{+} / b_{-}$. It is easy to check that

$$
f_{t}^{*} \Omega^{\prime}=\frac{\phi \circ f_{t}}{\phi} \Omega^{\prime}
$$

for all $t \in \mathbb{R}$. Thus for any $n$-dimensional parallelepiped $\Pi$ in a tangent space to $M$ and $t \in \mathbb{R}$, we have

$$
\|\Pi\|^{\prime} \leq L\left\|T f_{t}(\Pi)\right\|^{\prime}
$$

2.5. Lemma. If $\Phi=\left\{f_{t}\right\}$ is a volume preserving Anosov flow with constants defined in (1.1) and (1.2), then

$$
\left\|T f_{-t}(v \wedge w)\right\|^{\prime} \leq L(b c)^{n-3}\left(\mu_{+}^{n_{s}-1} \lambda_{+}^{n_{u}-1}\right)^{t}\|v \wedge w\|^{\prime},
$$

for all $v \in E^{s s}, w \in E^{u u}$ and $t \geq 0$.

Proof. Let $v \in E^{s s}, w \in E^{u u}$ and $t \geq 0$ be arbitrary. Set $v_{1}=T f_{-t}(v)$ and $w_{1}=T f_{-t}(w)$. Choose vectors $v_{2}, \ldots, v_{n_{s}} \in E^{s s}$ and $w_{2}, \ldots, w_{n_{u}} \in E^{u u}$ such that, relative to $\mathscr{R}^{\prime},\left(X, v_{1}, \ldots, v_{n_{s}}, w_{1}, \ldots, w_{n_{u}}\right)$ is an orthogonal basis of the corresponding tangent space and $v_{i}, w_{i}$ are all of unit length, for $i \geq 2$. 
Then:

$$
\begin{aligned}
\left\|T f_{-t}(v \wedge w)\right\|^{\prime} & =\left\|v_{1} \wedge w_{1}\right\|^{\prime} \\
& =\left\|X \wedge v_{1} \wedge \cdots \wedge v_{n_{s}} \wedge w_{1} \wedge \cdots \wedge w_{n_{u}}\right\|^{\prime} \\
& =\|\Pi\|^{\prime} \\
& \leq L\left\|T f_{t}(\Pi)\right\|^{\prime} \\
& =L\left\|T f_{t}\left(X \wedge v_{1} \wedge \cdots \wedge v_{n_{s}} \wedge w_{1} \wedge \cdots \wedge w_{n_{u}}\right)\right\|^{\prime} \\
& \leq L\left\|T f_{t}\left(v_{1} \wedge w_{1}\right)\right\|^{\prime}\left\|T f_{t}\left(X \wedge v_{2} \wedge \cdots \wedge v_{n_{s}} \wedge w_{2} \wedge \cdots \wedge w_{n_{u}}\right)\right\|^{\prime} \\
& \leq L\|v \wedge w\|^{\prime}(b c)^{n_{s}-1} \mu_{+}^{\left(n_{s}-1\right) t} \cdot(b c)^{n_{u}-1} \lambda_{+}^{\left(n_{u}-1\right) t} \\
& =L(b c)^{n-3} \mu_{+}^{\left(n_{s}-1\right) t} \lambda_{+}^{\left(n_{u}-1\right) t}\|v \wedge w\|^{\prime} .
\end{aligned}
$$

In the remainder of the paper we will always be working with $\mathscr{R}^{\prime}$ as the underlying Riemannian metric on $M$; the norms of tangent vectors and differential forms are taken relative to $\mathscr{R}^{\prime}$ and will be denoted by the symbol $\|\cdot\|$ (thus slightly abusing notation for the sake of keeping it less cumbersome).

\section{Proof of the Main theorem}

We will construct a smooth closed 1-form $\eta$ such that $u=\eta(X)>0$. Assuming for a moment that such a form has been found, the proof can be completed as follows. Define

$$
\tilde{X}=\frac{1}{u} X
$$

Then $\tilde{X}$ is an Anosov vector field [AS67] and $\eta(\tilde{X})=1$. Thus the Lie derivative of $\eta$ with respect to $\tilde{X}$ satisfies

$$
L_{\tilde{X}} \eta=\left(d i_{\tilde{X}}+i_{\tilde{X}} d\right) \eta=0
$$

which implies that $\eta$ is invariant with respect to the flow. It follows that its kernel $\operatorname{Ker}(\eta)$ is an invariant codimension one distribution transverse to the flow, so $\operatorname{Ker}(\eta)$ is forced to be the sum $\tilde{E}^{s u}=\tilde{E}^{s s} \oplus \tilde{E}^{u u}$ of the strong stable and strong unstable bundles of $\tilde{X}$. Since $\eta$ is closed, $\tilde{E}^{s u}$ is uniquely integrable, so by Plante [Pla72], $\tilde{X}$ admits a global cross section $\Sigma$, which is also a global cross section for $X$.

So it remains to construct a smooth 1-form $\eta$ with $\eta(X)>0$, which will be done in three steps. In the first two steps we construct a smooth 1 -form $\xi$ such that

$$
\xi(X)=1 \quad \text { and } \quad\|d \xi\|<1 .
$$

The third step consists of approximating $\xi$ by a smooth closed 1-form using Proposition 2.4.

Step 1. Let $\varepsilon>0$ be arbitrary. As in $\S 2.2$, for $\varepsilon<\varepsilon_{*}$, we can cover $M$ be smooth flow boxes $V_{1}^{\varepsilon}, \ldots, V_{\ell}^{\varepsilon}$, with $\ell$ independent of $\varepsilon$, such that with respect to the Hausdorff distance each $V_{i}^{\varepsilon}$ is close to a fixed open set $V_{i}$. In addition, we have smooth functions $\tau_{i}^{\varepsilon}: V_{i}^{\varepsilon} \rightarrow \mathbb{R}$ such that

$$
X \tau_{i}^{\varepsilon}=1, \quad \| d \tau_{i}^{\varepsilon}\left\lceil_{E^{s s}} \| \leq A \varepsilon^{\theta}, \quad \text { and } \quad\left\|d \tau_{i}^{\varepsilon}\right\| \leq B \varepsilon^{\theta-1},\right.
$$

where $A, B$ are constants independent of $\varepsilon$. 
Let $\left\{\psi_{i}^{\varepsilon}\right\}$ be a smooth partition of unity subordinate to the cover $\left\{V_{i}^{\varepsilon}\right\}$. Since $\ell$ and the sizes of the sets $V_{i}^{\varepsilon}$ are independent of $\varepsilon$, there is a constant $C>0$ also independent of $\varepsilon$ such that

$$
\sum_{i=1}^{\ell}\left\|d \psi_{i}^{\varepsilon}\right\| \leq C
$$

for all $0<\varepsilon<\varepsilon_{*}$. Define

$$
\xi_{0}^{\varepsilon}=\sum_{i=1}^{\ell} \psi_{i}^{\varepsilon} d \tau_{i}^{\varepsilon}
$$

3.1. Lemma. $\xi_{0}^{\varepsilon}$ is a smooth 1-form on $M$ with the following properties:

(a) $\xi_{0}^{\varepsilon}(X)=1$

(b) $\left\|\xi_{0}^{\varepsilon} \uparrow_{E^{s s}}\right\| \leq A \varepsilon^{\theta}$, for all $0<\varepsilon<\varepsilon_{*}$;

(c) $\left|d \xi_{0}^{\varepsilon}(v, w)\right| \leq D \varepsilon^{\theta}$, for all unit vectors $v, w \in E^{c s}$ and $0<\varepsilon<\varepsilon_{*}$, where $D$ is a constant independent of $\varepsilon$;

(d) $\left\|d \xi_{0}^{\varepsilon}\right\| \leq K \varepsilon^{\theta-1}$, for every $0<\varepsilon<\varepsilon_{*}$, where $K$ is a constant independent of $\varepsilon$.

Proof. Part (a) is clear. Part (b) follows easily from the second inequality in (3.1). To prove (c), first note that if $\beta$ is a bilinear form on an inner product space $E$ which splits into two orthogonal subspaces $E_{1}$ and $E_{2}$ and $\beta_{i j}=\beta \uparrow_{E_{i} \times E_{j}}$, then

$$
\|\beta\| \leq\left\|\beta_{11}\right\|+2\left\|\beta_{12}\right\|+\left\|\beta_{22}\right\| .
$$

We fix $p \in M$ and take $\beta=d_{p} \xi_{0}^{\varepsilon}, E_{1}=E_{p}^{c}$ and $E_{2}=E_{p}^{s s}$. Since

$$
d \xi_{0}^{\varepsilon}=\sum_{i=1}^{\ell} d \psi_{i}^{\varepsilon} \wedge d \tau_{i}^{\varepsilon}
$$

if $v, w \in E^{s s}$ are unit vectors, then

$$
\left|d \xi_{0}^{\varepsilon}(v, w)\right| \leq 2 C \varepsilon^{\theta}
$$

and

$$
\left|d \xi_{0}^{\varepsilon}(X, v)\right|=\left|\sum_{i} d \psi_{i}^{\varepsilon}(X) d \tau_{i}^{\varepsilon}(v)-d \psi_{i}^{\varepsilon}(v) d \tau_{i}^{\varepsilon}(X)\right|=\left|\sum_{i} d \psi_{i}^{\varepsilon}(X) d \tau_{i}^{\varepsilon}(v)\right| \leq A C \varepsilon^{\theta}
$$

where we used $d \tau_{i}^{\varepsilon}(X)=1$ and $\sum_{i} d \psi_{i}^{\varepsilon}(v)=d\left(\sum_{i} \psi_{i}^{\varepsilon}\right)(v)=0$. Thus by (3.3) we can take $D=(A+2) C \varepsilon$ in (c). Finally, (3.2) and $\left\|d \tau_{i}^{\varepsilon}\right\| \leq B \varepsilon^{\theta-1}$ imply

$$
\left\|d \xi_{0}^{\varepsilon}\right\|=\left\|\sum_{i=1}^{\ell} d \psi_{i}^{\varepsilon} \wedge d \tau_{i}^{\varepsilon}\right\| \leq B C \varepsilon^{\theta-1}
$$

so (d) holds with $K=B C$.

In summary, for every $0<\varepsilon<\varepsilon_{*}$ we have a 1-form which is small on $E^{s s}$, whose exterior derivative is small when restricted to $E^{c s}$, but whose overall norm grows as $\varepsilon^{\theta-1}$, as $\varepsilon \rightarrow 0$. 
Step 2. To remedy the problem represented by part (d) of the previous lemma, we flow backwards and use Lemma 2.5. Let $t>0$ be large (how large will be specified shortly) and set

$$
\xi_{t}^{\varepsilon}=f_{-t}^{*} \xi_{0}^{\varepsilon}
$$

3.2. Lemma. There exists a constant $H>0$ independent of $\varepsilon$ and $t$ such that

$$
\left\|d \xi_{t}^{\varepsilon}\right\| \leq \max \left\{H \varepsilon^{\theta-1}\left(\mu_{+}^{n_{s}-1} \lambda_{+}^{n_{u}-1}\right)^{t}, H \varepsilon^{\theta} \mu_{-}^{-2 t}\right\}
$$

Proof. Observe that if $\beta$ is a bilinear form as in the proof of the previous lemma and $\left\|\beta_{22}\right\| \leq\left\|\beta_{i j}\right\|$, for all $i, j=1,2$, then

$$
\|\beta\| \leq 4 \max \left(\left\|\beta_{11}\right\|,\left\|\beta_{12}\right\|\right) .
$$

Fix $p \in M$ and take $\beta=d_{p} \xi_{t}^{\varepsilon}, E=T_{p} M, E_{1}=E_{p}^{c s}$ and $E_{2}=E_{p}^{u u}$. Since $d \xi_{t}^{\varepsilon}=f_{-t}^{*}\left(d \xi_{0}^{\varepsilon}\right)$ and $\left\|T f_{-t} \uparrow_{E^{c s}}\right\| \leq c \mu_{-}^{-t}$, for $t>0$, Lemmas 3.1 and 2.5 imply

$$
\left\|\beta_{11}\right\| \leq c^{2} D \varepsilon^{\theta} \mu_{-}^{-2 t} \quad \text { and } \quad\left\|\beta_{12}\right\| \leq K L \varepsilon^{\theta-1}(b c)^{n-3}\left(\mu_{+}^{n_{s}-1} \lambda_{+}^{n_{u}-1}\right)^{t} .
$$

Note that the second inequality holds because if $v \in E^{c s}$ and $w \in E^{u u}$, then $\left|d \xi_{t}^{\varepsilon}(v, w)\right|=$ $\left|d \xi_{0}\left(T_{-t}(v \wedge w)\right)\right|$ is largest if $v \in E^{s s}$. Observe also that $\left\|\beta_{22}\right\|$ is smaller than $\left\|\beta_{i j}\right\|(i, j=1,2)$ since the flow contracts strong unstable vectors in negative time.

The proof of the lemma is now complete with $H=4 \max \left(c^{2} D, K L(b c)^{n-3}\right)$.

Since $\xi_{t}^{\varepsilon}(X)=1$, for all $0<\varepsilon<\varepsilon_{*}$ and $t>0$, the following lemma will complete the construction of the desired form $\xi$.

3.3. Lemma. There exist $0<\varepsilon<\varepsilon_{*}$ and $t>0$ such that $\left\|d \xi_{t}^{\varepsilon}\right\|<1$.

Proof. By the previous Lemma, it suffices to show that the following system of inequalities

$$
\begin{aligned}
H \varepsilon^{\theta-1}\left(\mu_{+}^{n_{s}-1} \lambda_{+}^{n_{u}-1}\right)^{t} & <1 \\
H \varepsilon^{\theta} \mu_{-}^{-2 t} & <1
\end{aligned}
$$

admits a solution $\varepsilon, t>0$. Solving the first inequality for $t$ we obtain

$$
t>\frac{(1-\theta) \log \varepsilon-\log H}{\left(n_{s}-1\right) \log \mu_{+}+\left(n_{u}-1\right) \log \lambda_{+}} .
$$

The second inequality is equivalent to

$$
t<\frac{\log H+\theta \log \varepsilon}{2 \log \mu_{-}}
$$

There exists $t$ with these properties if and only if we can find $0<\varepsilon<\varepsilon_{*}$ such that

$$
\frac{(1-\theta) \log \varepsilon-\log H}{\left(n_{s}-1\right) \log \mu_{+}+\left(n_{u}-1\right) \log \lambda_{+}}<\frac{\log H+\theta \log \varepsilon}{2 \log \mu_{-}} .
$$

Since $H$ is fixed and $\varepsilon$ is small, this is possible if

$$
\frac{(1-\theta) \log \varepsilon}{\left(n_{s}-1\right) \log \mu_{+}+\left(n_{u}-1\right) \log \lambda_{+}}<\frac{\theta \log \varepsilon}{2 \log \mu_{-}}
$$

which is equivalent to (1.3). 
Step 3. Choose $0<\varepsilon<\varepsilon_{*}$ and $t>0$ such that $\left\|d \xi_{t}^{\varepsilon}\right\|<1$ and set

$$
\xi=\xi_{t}^{\varepsilon}
$$

By Proposition 2.4 there exists a smooth closed 1-form $\eta$ such that

$$
\|\xi-\eta\| \leq\|d \xi\|<1
$$

Since $\xi(X)=1$, it follows that $\eta(X)>0$. This completes the construction of $\eta$ and concludes the proof of the theorem.

\section{REFERENCES}

[Ano67] Dimitri V. Anosov, Geodesic flows on closed Riemannian manifolds of negative curvature, Proc. Steklov Math. Inst. 90 (1967), AMS Translations (1969).

[Arm82] Pedro Armendariz, Codimension one Anosov flows on manifolds with solvable fundamental group, Ph.D. thesis, Universidad Autónoma Metropolitana, Unidad Iztapalapa, Mexico, D. F., 1982.

[AS67] Dimitri V. Anosov and Yakov G. Sinai, Some smooth ergodic systems, Russ. Math. Surveys 22 (1967), 103-167.

[Asa08] Masayuki Asaoka, On invariant volumes of codimension-one Anosov flows and the Verjovsky conjecture, Invent. Math. 174 (2008), no. 2, 435 - 462.

[BG09] Christian Bonatti and Nancy Guelman, Transitive Anosov flows and Axiom-A diffeomorphisms, Ergod. Th. Dynam. Syst. 29 (2009), 817-848.

[Eva98] Lawrence C. Evans, Partial differential equations, Graduate Studies in Mathematics, vol. 19, AMS, 1998.

[Fra70] John Franks, Anosov diffeomorphisms, Proc. of Symposia in Pure Math., 1970, pp. 61-93.

[Ghy89] Etienne Ghys, Codimension one Anosov flows and suspensions, Lecture Notes in Mathematics, vol. 1331, pp. 59-72, Springer Verlag, 1989.

[Has94] Boris Hasselblatt, Regularity of the Anosov splitting and of horospheric foliations, Ergodic Theory Dynam. Systems (1994), 645-666.

[Has97] _ Regularity of the Anosov splitting II, Ergodic Theory Dynam. Systems (1997), 169-172.

[HPS77] Morris W. Hirsch, Charles C. Pugh, and Michael Shub, Invariant manifolds, Lecture Notes in Mathematics, vol. 583, Springer-Verlag, Berlin-New York, 1977.

[HW99] Boris Hasselblatt Hasselblatt and Amie Wilkinson, Prevalence of non-Lipschitz Anosov foliations, Erg. Th. Dyn. Syst. 19 (1999), no. 3, 643-656.

[KH95] Anatole Katok and Boris Hasselblatt, Introduction to the modern theory of dynamical systems, Encyclopedia of Mathematics and its Applications, vol. 54, Cambridge University Press, 1995.

[New70] Sheldon Newhouse, On codimension one Anosov diffeomorphisms, Amer. J. of Math. 92 (1970), 761-770.

[Pla72] Joseph Plante, Anosov flows, Amer. J. of Math. 94 (1972), 729-754.

[Pla81] _ Anosov flows, transversely affine foliations, and a conjecture of Verjovsky, J. London Math. Soc. 2 (1981), 359-362.

[Pla83] Solvable groups acting on the line, Trans. Amer. Math. Soc. 278 (1983), no. 1, $401-414$.

[PSW97] Charles C. Pugh, Michael Shub, and Amie Wilkinson, Hölder foliations, Duke Math. J. 86 (1997), no. 3, 517-546.

[Sch57] Saul Schwartzman, Asymptotic cycles, Annals of Math. 66 (1957), 270-284.

[Sha93] Richard Sharp, Closed orbits in homology classes for Anosov flows, Ergod. Th. Dynam. Syst. 13 (1993), 387-408.

[Sim96] Slobodan N. Simić, Lipschitz distributions and Anosov flows, Proc. Amer. Math. Soc. 124 (1996), no. 6, 1869-1877.

[Sim97]_ Codimension one Anosov flows and a conjecture of Verjovsky, Ergod. Th. Dynam. Syst. 17 (1997), 1211-1231.

[Spi05] Michael Spivak, A Comprehensive Introduction to Differential Geometry, 3rd ed., vol. 1, Publish or Perish, 2005 .

[Ste70] Elias M. Stein, Singular integrals and differentiability properties of functions, Princeton University Press, 1970.

[Ver74] Alberto Verjovsky, Codimension one Anosov flows, Bol. Soc. Matematica Mexicana 19 (1974), 49-77. 
Department of Mathematics and Statistics, San José State University, San José, CA 95192-0103

E-mail address: simic@math.sjsu.edu 